\title{
Skin test development in leprosy: progress with first-generation skin test antigens, and an approach to the second generation
}

\author{
P. J. BRENNAN \\ Department of Microbiology, Colorado State University, \\ Fort Collins, Colorado, USA
}

\begin{abstract}
Summary One of the most urgent needs from leprosy research is a test for infection. The lepromin test is not suitable as a diagnostic test for leprosy, and neither the Rees nor the Convit soluble antigens has appeared sufficiently specific. Because two new antigens, MLSA-LAM and MLCwA, may not fully meet the requirements for specificity, we have embarked upon the preparation of a second generation of skin test antigens. Size-fractionated cryptozoic proteins were prepared from $M$. leprae by electroelution from preparative sodium dodecylsulphate-polyacrylamide gel electrophoresis, and individual fractions were probed with polyclonal and monoclonal antibody reagents to identify both known and novel proteins. In addition, immunological responses were assessed in M. leprae-sensitized guinea pigs against both crude subcellular fractions (cytosol, membrane, and soluble cell wall proteins) and the size-fractionated cytosolic proteins. A particularly promising subcellular fraction is the membrane fraction of $M$. leprae, which contains many proteins unique to the organism. Clinical trials of the $M$. leprae membrane proteins are now being planned.
\end{abstract}

The goal of eliminating leprosy as a serious public health problem, as originally defined by the World Health Assembly, is probably feasible in the short term. However, it is now obvious that we do not have the tools to achieve the goal of a 'World without Leprosy.' That new-case detection rates in highly endemic countries have remained stable over the years has been attributed to enhanced surveillance or an extraordinarily long incubation period, so that the reduction of prevalence has not had an obvious impact on incidence. One of the greatest needs from leprosy research is definitive, diagnostic tools that permit recognition of exposure, i.e. a test for infection, that may be applied selectively in endemic communities. After a generation of research and application, the failure of serological and gene amplification techniques to fill this need has proved disappointing. In addition, in light of the evidence that household contacts of leprosy patients are four to six times more likely than non-contacts to develop leprosy, there is an urgent need to broaden further the definition and identification of high-risk groups, in order to render more effective the application of prophylactic measures.

The immune response to mycobacterial infections, both leprosy and tuberculosis, is predominantly cellular, ${ }^{1}$ and examination of the delayed-type hypersensitivity response, one aspect of cellular immunity, has proved to be a convenient, cost-effective test for 'exposure' to $M$. tuberculosis ${ }^{2}$ (the term 'exposure' is misleading; it is, in fact, a code word for infection). Foremost among these tests is the Mantoux test, which is based on M. tuberculosis tuberculin or purified protein derivative (PPD). ${ }^{2}$ The test is very effective in monitoring 
infection in populations with low endemicity and no BCG vaccination programme, as in the majority of communities in the USA and Canada. However, it is beset with problems: (i) low specificity in populations with high endemicity of mycobacterial infections in general, because of cross-reactivity of the antigenic components of PPD among mycobacterial species; (ii) variability in reading the test; (iii) the need for patients to return after $48-72 \mathrm{~h}$ for the reading; and (iv) modulation of the response to the skin test by underlying illness or immunosuppression. ${ }^{2,3}$ The specificity of a positive PPD skin test has been reported to exceed $95 \%$, although the sensitivity of the test may be less than $75 \%{ }^{4}$

Nevertheless, the ease and low cost of the tuberculin skin test and its effectiveness in certain settings enhance the attractiveness of skin tests for leprosy. The concept is old. In 1919, Mitsuda reported that some human subjects, including leprosy patients, injected with a suspension of tissue from lepromatous patients, developed a local nodular reaction. ${ }^{5}$ Subsequently, successive improvements have been made, resulting in a more refined test, known generally as a lepromin test, which is widely used for both classification and prognosis in leprosy. 6,7

Two responses to the lepromin test have been described: (i) an early response, known as the Fernandez reaction, ${ }^{8}$ which is measured between 24 and $72 \mathrm{~h}$; and (ii) a late response, known as the Mitsuda reaction, ${ }^{6}$ which is measured between 3 and 4 weeks after testing. ${ }^{9}$ The Fernandez reaction is thought to be similar to the tuberculin reaction, in that it measures the subject's DTH response to the soluble M. leprae antigens. ${ }^{10}$ The Mitsuda reaction is considered to be an induced hypersensitivity granuloma, ${ }^{7,11}$ in response to soluble $M$. leprae antigens, possibly after they have been processed and released by antigen-presenting cells. Patients with leprosy respond in a characteristic way to the lepromin test: ${ }^{7,12}$ lepromatous patients do not respond to lepromin, whereas patients at the tuberculoid pole of the leprosy spectrum show both positive Fernandez and Mitsuda responses. However, Mitsuda himself observed that healthy individuals responded to his test, ${ }^{6}$ a point since confirmed and elaborated; healthy individuals who live in non-endemic countries or in countries of low endemicity are Mitsuda-positive but Fernandez-negative. ${ }^{12}$ This phenomenon of unexpectedly large proportions of Mitsuda responders among healthy individuals has led to speculation that the lepromin test is actually a form of vaccination. ${ }^{12}$ Because the Fernandez reaction is usually negative in normal populations in non-endemic areas, but is also frequently negative in leprosy patients, whereas the Mitsuda reaction is positive in both healthy subjects and many leprosy patients, the lepromin test is not considered to be suitable as a diagnostic test for leprosy, and is useful only in the classification of established leprosy.

Because of the difficulty of obtaining human biopsy specimens containing sufficient $M$. leprae, lepromin prepared from human tissues was replaced by lepromin derived from infected armadillos. In addition, both Rees and Convit developed soluble antigen preparations from armadillo-derived $M$. leprae ${ }^{13}$ Rees' soluble antigen, MLSA, has been studied more thoroughly. Skin test studies were carried out among close contacts of multibacillary (MB) leprosy patients at three leprosy centres in India, and among casual contacts of the disease at two centres. ${ }^{14}$ At all three centres, close contact with patients with MB leprosy was associated with early acquisition of skin test positivity to MLSA (known also as leprosin A), reaching $70 \%$ positivity by age 15 years. Among casual contacts, skin test positivity to MLSA was significantly less frequent, reaching $40 \%$ by age 15 .

Another, large study ${ }^{15}$ among 2607 individuals, consisting of 207 leprosy patients, 476 household contacts, and 1924 people who resided in non-case households, in which both the Convit and Rees antigens were applied, concluded that 'Rees and Convit antigens are not 
useful in the identification of $M$. leprae infection or in the confirmation of leprosy diagnosis in a leprosy-endemic population with a high prevalence of non-specific sensitivity.' This study resulted in considerably lessened interest in use of the Rees and Convit antigens for diagnosis.

On the other hand, extensive work with these antigens in Northern Malawi has yielded more promising results. ${ }^{16}$ Tests among biopsy-confirmed patients, household contacts, and individuals with no known contact provided measures of the sensitivity and specificity of these skin tests for infection with $M$. leprae. Although induration was, in general, not sharply defined, and although the frequency distribution of the diameter of induration appeared to differ between populations, a clear bimodality of the diameter of induration and an increasing prevalence of skin test positivity with age suggested that these skin tests were specific for some mycobacterial exposure. A number of workers have concluded that there is need for improvement of methods of preparative, standardization, sensitivity, and specificity. ${ }^{15,16}$

With these challenges in mind, we undertook the preparation of a new generation of skintest antigens in the early 1990s, working under the stringent conditions prescribed by the US Code of Federal Regulations, and supervised by the US Food and Drug Administration (FDA). When we first applied for an investigational new drug exemption (IND) for new skin test antigens in 1993, the FDA ruled that we had not fully met their requirements. During the years 1993-1998, we built a Pilot Plant Facility for production of the antigens, and developed protocols and standard operating practices for manufacture of the antigens, assessment of their safety, testing their pharmacological and toxicological properties, qualitative analysis of their composition, testing their potency both in vivo and in vitro, packaging, and sterility testing.

These protocols and practices were applied to two new antigen preparations prepared from armadillo-derived $M$. leprae.$^{17}$ The first we named MLSA-LAM, to show its relationship to the Rees soluble antigen. However, much of the large quantities of lipoarabinomannan (LAM) and lipids present in a low-speed $(27,000 \mathrm{~g})$ supernatant from a sonicated preparation of $M$. le prae was removed by extraction with detergent. The second product, $M$. leprae cell wall antigen (MLCwA), was derived by extraction of the cell walls (the 27,000 $g$ pellet) with $2 \%$ sodium dodecylsulphate (SDS), followed by removal of the SDS by column chromatography. The products were potent when assayed for skin test response in $M$. leprae-sensitized guinea pigs: at $48 \mathrm{~h}, 0.5 \mu \mathrm{g}$ or more MLSA-LAM induced induration greater than $5 \mathrm{~mm}$ in diameter and $0 \cdot 1 \mu \mathrm{g}$ MLCwA produced a similar degree of induration. Much larger doses of these materials were required to elicit responses in $M$. tuberculosis-sensitized guinea pigs.

In the majority of patients whose cells responded to $M$. leprae, both MLSA-LAM and MLCwA strongly induced proliferation of lymphocytes. ${ }^{18}$ Application of the IFN $\gamma$ assay to these products also confirmed their potency and partial specificity. ${ }^{19}$ Twenty-four hour whole blood assays in Nepal showed that the T cells of both paucibacillary leprosy patients and the household contacts of leprosy patients produce significantly greater amounts of IFN $\gamma$ in response to stimulation by MLCA-LAM or MLCwA than do the T cells of others residing in endemic communities. Stimulated by the same antigens, the $\mathrm{T}$ cells of tuberculosis patients produced smaller quantities of IFN $\gamma$, suggesting a lesser degree of cross-reactivity with $M$. tuberculosis antigens than that demonstrated by Rees' MLSA.

In 1998, the FDA approved a phase I trial of the clinical safety of these fractions among 10 volunteers in Fort Collins, Colorado. The results showed that these fractions are non-toxic, and do not elicit a skin test reaction among individuals from non-endemic countries who have not been exposed to M. leprae. A phase II trial under the direction of Drs Ruth Butlin and Paul Roche, Anandaban Leprosy Hospital, Kathmandu, Nepal, to investigate the appropriate 
dosage, immunological dynamics, sensitivity, and specificity of the fractions in a country in which leprosy is highly endemic, planned originally for the year 2000, is to be carried out in $2001 .^{20}$

The planning and undertaking of clinical trials that involve American investigators or materials produced in the USA is very demanding, requiring approval by the funding agency, the FDA, the Office of Human Research Protection, and the local institutional committee that reviews research on human subjects. A detailed protocol, consent forms in English, in the native language(s), and back-translations from the native languages, case-report forms, standard operating practices (SOP) with respect to key functions, and a brochure for participating investigators all must be prepared. In addition, the products must be marked in code with a specified label format, and prepared for shipping with temperature monitors. In fact, the procedures that must be followed in order to obtain approval of a skin test antigen to be used in man are as stringent as those for a new vaccine. These onerous, time-consuming, and very demanding tasks are not be regarded as burdens imposed by bureaucrats, but rather as necessary safeguards for human subjects, which must be pursued with thoroughness and complete transparency. The burden is particularly great in an area such as leprosy, because the available resources are insufficient to employ professionals who are well versed in these tasks.

Because these fractions may not fully meet the requirements for specificity, we are already preparing second-generation skin test antigens. One approach that has been used to dissect the cell-mediated immune response to a very complex mixture of proteins is electroelution of size-fractionated proteins from preparative sodium dodecysulphatepolyacrylamide electrophoresis (SDS-PAGE) gels. We used this technique to produce sizefractionated cryptozoic proteins from $M$. leprae. The individual fractions were probed with polyclonal and monoclonal antibody reagents by ELISA and Western blot to identify known proteins, and, through the process of elimination, to identify novel proteins, reinforced by N-terminal sequencing of two-dimensional gel spots. In addition, immunological responses (both antibody-mediated and delayed-type hypersensitivity responses) were assessed in M. leprae-sensitized guinea pigs against both crude subcellular fractions (cytosol, membrane, and soluble cell wall proteins) and size-fractionated cytosolic proteins. Thus, we have generated a detailed portrait of the protein profile and immunogenicity of the subcellular fractions, and further characterized additional novel proteins that comprise the subcellular fractions of M. leprae.

One particularly promising subcellular fraction is the membrane fraction of $M$. leprae. This fraction may have been excluded by the filtration process from Rees' MLSA; in the case of MLSA-LAM, membrane proteins were largely extracted by the two-phase detergent step required to remove LAM. We have already shown that membranes contain many proteins unique to $M$. leprae, and that this fraction is much more active in $M$. leprae-sensitized guinea pigs than in those sensitized with $M$. tuberculosis. Clinical trials of the M. leprae membrane proteins are now being planned.

The new information on protein composition arising from sequencing the genome of $M$. leprae, especially with respect to those proteins that are specific to M. leprae, offers great promise for new diagnostic reagents. A vexing problem and challenge is the recognition that $M$. leprae recombinant proteins expressed in $E$. coli do not reflect the immunogenicity of the parent proteins. Procedures must be developed to ensure that the recombinant products reflect the folding patterns and other post-translational modifications of the parent compounds. 


\section{References}

1 Daniel T. The immunology of tuberculosis. Clin Chest Med, 1980; 1: 189-201.

2 Snider D. The tuberculosis skin test. Am Rev Respir Dis, 1982; 125S: 108-118.

${ }^{3}$ Daniel TM, Ellner JJ. Immunology of tuberculosis. In: Reichman L, Hershfield ES (eds) Tuberculosis. A comprehensive international approach, 1st edition. Marcel Dekker, New York, 1993, pp 75-101.

${ }^{4}$ Frances J, Seiler RJ, Wekie IW et al. The sensitivity and specificity of various tuberculin tests using bovine PPD and other tuberculins. Vet Rec, 1978; 103: 420-425.

5 Mitsuda K. On the value of a skin reaction to a suspension of leprosy nodules. Jpn J Dermatol Urol, 1919; 19: 697-708.

${ }^{6}$ Mitsuda K. On the value of a skin reaction to a suspension of leprous nodules. Int J Lepr, 1953; 21: 347-358.

${ }^{7}$ Lefford MJ. Lepromin as an indicator and inducer of protective immunity. Lepr Rev, 1981; 52 Suppl: 221-229.

${ }^{8}$ Fernandez JMM. The early reaction induced by lepromin. Int J Lepr, 1940; 8: 1-15.

9 Gill HK, Mustafa AS, Godal T. Induction of delayed hypersensitivity in human volunteers immunized with a candidate leprosy vaccine consisting of killed Mycobacterium leprae. Bull WHO, 1986; 64: 121-126.

${ }^{10}$ Lowe J, MacNulty F. Tuberculosis and leprosy; immunological studies. Lepr Rev, 1953; 24: 61-90.

11 Stanford JL. Skin testing with mycobacterial reagents in leprosy. Tubercle, 1984; 65: 63-74.

12 Gill HK, Mustafa AS, Godal T. Vaccination of human volunteers with heat-killed M. leprae: local responses in relation to the interpretation of the lepromin reaction. Int $J$ Lepr, 1988; 56: 36-44.

13 Anonymous. Vaccination trials against leprosy. Meeting of the Epidemiology Subgroup of the Scientific Working Group on the Immunology of Leprosy, Geneva, 11-13 February 1985, TDR/IMMLEP/EPD/85.3, pp. 7-8.

14 Lord R, Naish C, Taylor C et al. Skin test studies on close contacts of leprosy patients in India. Int J Lepr, 1989; 57: $801-809$.

15 Gupte MD, Anantharaman DS, Nagaraju B et al. Experiences with Mycobacterium leprae soluble antigens in a leprosy endemic population. Lepr Rev, 1990; 61: 132-144.

16 Fine PEM, Ponnighaus JM, Rees RJW et al. Epidemological studies with M. leprae soluble antigens. Int J Lepr, 1984; 52 (Suppl): 692 (abstract).

17 Brennan PJ, TerLouw SM. New skin test antigens for the diagnosis of leprosy. Transactions of the 19th International Leprosy Congress. Int J Lepr, 1998; 66: 96A (abstract).

18 Weir RE, Brennan PJ, Butlin CR, Dockrell HM. Use of a whole blood assay to evaluate in vitro T cell responses to new leprosy skin test antigens in leprosy patients and healthy subjects. Clin Exp Immunol, 1999; 116: 263-269.

19 Manandhar R, LeMaster JW, Butlin CR et al. Interferon-gamma responses to candidate leprosy skin test reagents detect exposure to leprosy in an endemic population. Int J Lepr, 2000; 68: 40-48.

${ }^{20}$ LeMaster JW, Roche P. Are we any closer to being able to measure leprosy exposure? Lepr Rev, 2000; 71: 2-4. 